\title{
It's time to focus on decreasing cardiovascular mortality in NAFLD population: potential use of statins and PCSK9 inhibitors.
}

\author{
Tarek Ajam*1, Anuj Chhaparia1, Zachary Oman', Ali Mehdirad ${ }^{2}$ \\ ${ }^{1}$ Department of Internal Medicine, Saint Louis University Saint Louis, MO, USA \\ ${ }^{2}$ Division of Cardiology, Saint Louis University, Saint Louis, MO, USA
}

\begin{abstract}
With the alarming rise in prevalence of nonalcoholic fatty liver disease (NAFLD) in the world, there is an increasing need to recognize the strong association between NAFLD and cardiovascular disease (CVD). NAFLD is dynamically involved in the pathogenesis of CVD through the processes that include increased oxidative stress, endothelial dysfunction, accelerated progression of atherosclerosis, and irregular fatty acid, glucose, and lipoprotein metabolism. By reviewing and understanding the mechanisms, the aim of this review is to introduce the association between NAFLD and CVD to health care providers and the importance of statin therapy and mortality benefits. More importantly, we discuss the role of statins as a treatment strategy and possible future directions.
\end{abstract}

Keywords Nonalcoholic fatty liver disease, Cardiovascular disease, Mortality, Statin, PCSK 9 inhibitor. Accepted on September 18, 2017

\section{Introduction}

Nonalcoholic fatty liver disease (NAFLD), considered the most common liver disorder in Western countries, is estimated to affect approximately one-quarter of the general population $[1,2]$. The prevalence of NAFLD matches the increasing rates of type 2 diabetes mellitus and obesity worldwide. In addition, there are up to $95 \%$ of obese persons and $75 \%$ of diabetics likely to have NAFLD [3]. NAFLD is a slowly progressive disease which covers a spectrum of hepatic pathologies, ranging from simple steatosis, variable grades of fibrosis, and nonalcoholic steatohepatitis (NASH). Lipid accumulation is the major hallmark with fatty infiltrates affecting more than $5 \%$ of hepatocytes [4].

\section{Pathogenesis}

There is a significant association between NAFLD and cardiovascular disease (CVD) as multiple studies have reported increased adverse cardiovascular events in NAFLD patients compared with the general population [5-13]. A recent metaanalysis with 27 cross-sectional studies demonstrated that NAFLD was associated with markers of subclinical atherosclerosis, such as increased carotid artery intimal-medial thickness, impaired flow-mediated vasodilation, or increased coronary artery calcification [14]. In a prospective study, the 14-year risk of mortality from cardiovascular causes was twofold in patients with biopsy-proven NAFLD where the primary cause of death was CVD rather than liver disease [15].

Currently, the understanding of the pathophysiological pathways linking NAFLD to CVD remains elusive. Although mechanisms such as oxidative stress, abdominal obesity and insulin resistance have been considered [16]. The liver is central to regulation of systemic inflammation and is acted upon numerous inflammatory factors. There is evidence that suggests NAFLD exacerbates hepatic and peripheral insulin resistance predisposing subjects to atherogenic dyslipidemia while releasing pro-inflammatory, vasoactive, and thrombogenic factors that instigate the development of CVD [17-20]. NAFLD patients have more systemic inflammation and have higher risk of thrombosis than that of the general population as evidenced by higher levels of oxidative stress and inflammation [21]. Several studies have demonstrated increased coronary atherosclerotic plaque burden in the presence of NAFLD [22-24].

A known marker of adverse cardiovascular outcomes, Creactive protein, is significantly elevated in NAFLD patients compared to non-NAFLD group [25]. C-reactive protein is a predictor of cardiovascular events in several studies [26]. The severity of NAFLD is a direct predictor of worsening inflammatory and insulin resistance. A second known marker of adverse cardiovascular outcomes is gammaglutamyltransferase (GGT) level. GGT has a role in oxidative stress and is expressed in atherosclerotic plaques. Several studies have shown an association between GGT levels and cardiovascular mortality $[9,10,27]$ including a meta-analysis of relationship of GGT and adverse cardiovascular events such as myocardial infarction [11].

Excessive free fatty acid supply leads to cardiac lipotoxicity by triggering intracellular lipid accumulation and consuming cardiomyocyte oxidative capacity. This results in increased oxidative stress and subsequent cardiomyocyte dysfunction and apoptosis [28,29]. Adverse alterations in fatty acid, glucose, and lipoprotein metabolism seen in NAFLD leads to the progression of insulin resistance [30] and overall dysfunctional metabolic state [31].

NAFLD has also been found to affect carotid artery disease and incidence of cerebrovascular accidents via direct effects of plasminogen activator inhibitor-1 (PAI-1) [32-35]. PAI-1 is produced by hepatic tissue and when fatty infiltration is 
Citation: Tarek A, Anuj C, Zachary $O$, et al. It's time to focus on decreasing cardiovascular mortality in NAFLD population: potential use of statins and PCSK9 inhibitors. Curr Trend Cardiol. 2017;1(2):\$\{pages\}.

present, less PAI-1 is produced creating a pro-thrombotic state and higher prevalence of cerebral vascular accidents secondary to carotid atherosclerotic plaques and luminal narrowing [33]. One study showed significant association between NAFLD and carotid intima-media thickness with an estimated $13 \%$ increase in carotid intima-media thickness for NAFLD cases compared with controls [36].

Patients who have NAFLD also have reduced concentrations of adiponectin. Adiponectin is a key element in lipid management because it activates AMP-activated protein kinase and peroxisome proliferator-activated receptor- $\alpha$ which subsequently leads to elevated triglycerides, denser LDL particles, and reduced levels of HDL cholesterol. Additionally, there are qualitative alterations present in the HDL which further limits its athero-protective benefit and CVD prevention [37].

The current recommended management strategies for NAFLD and CVD prevention include first correcting insulin resistance and hyperinsulinemia by reducing fat mass, in particular visceral adiposity. This can be accomplished through weight loss, physical exercise, and diet and lifestyle modifications. An additional strategy is to reverse or prevent further hepatocellular damage by inhibiting lipid peroxidation and oxidative stress using hepatoprotective agents such as antiinflammatory or anti-apoptotic agents [38].

\section{Statins}

Due to the association between NAFLD and CVD, lipidlowering agents, such as statins, may be beneficial because of their ability to significantly reduce atherogenesis and inflammation. The benefit of statins in primary and secondary prevention of cardiovascular disease is well described. However, adverse effects of statin therapy, such as myalgias and transaminitis, create an obstacle for health care providers when attempting adequate cholesterol control.

The current guidelines for cholesterol management in patients with an ASCVD risk score of less than $7.5 \%$ would recommend against initiating statin therapy [39]. However, because of the known risk with NAFLD, not previously considered in current guidelines, statin therapy would likely prove beneficial.

The use of statin medications has been accompanied by concern for hepatotoxicity. The primary mechanism of action for statin therapy is to inhibit the central pathway of 3hydroxy-3-methylglutaryl coenzyme A (HMG-CoA) reductase. They also initiate activation of Sterol Regulatory Element Binding Proteins (SREBPs) which subsequently increase the number of hepatic LDL receptors and serum lipid degradation. In addition, peroxisome proliferator-activated receptor gamma (PPAR $\gamma)$ within macrophages are activated via cycloxygenase 2-mediated reaction which promote fatty acid oxidation reducing the triglyceride supply for VLDL synthesis [40,41].

Recent studies have not demonstrated an increase in serum aminotransferase level with use of statins [42]. A meta-analysis of 49,275 patients with hyperlipidemia and NAFLD treated with statins with baseline elevated liver enzymes demonstrated no significant changes in liver biochemistry on statins [43].

The effect of statin therapy on hepatic histology in NAFLD is not completely understood. Tumor necrosis factor and interleukin-6 serum markers are associated with histologic changes in advanced Non-Alcoholic Steatohepatitis (NASH). These markers have been shown to decrease with statin therapy [44]. In a case control study by Ekstedt et al. NAFLD patients who received statins as treatment for hyperlipidemia or cardiovascular disease had lower progression rates of liver steatosis than those without statin therapy [45].

A three-year prospective study (GREACE) demonstrated a therapeutic benefit of statin therapy (Atorvastatin) on clinical endpoints in NAFLD patients with established coronary heart disease. $89 \%$ of patients in the statin therapy group with abnormal liver tests at baseline demonstrated normalized liver enzyme levels while the patients not on statin therapy experienced progressive worsening of liver enzymes [46]. There was also a $49 \%$ relative risk reduction of cardiovascular events between statin therapy group compared to non-statin therapy group, and overall risk of cardiovascular events was reduced by $68 \%$ in patients with abnormal liver tests. The statin therapy group demonstrated both reduction of cardiovascular risk and improvement in abnormal liver tests which may prevent the progression of NAFLD.

Patients with NAFLD have approximately a two-fold increased risk of cardiovascular mortality compared with age-matched general population [8]. With increased cardiovascular risk associated with NAFLD as a result of pro-atherogenic and proinflammatory states, it should be clear that an antiatherosclerotic and pleiotropic agent would be of great benefit in reducing cardiovascular risk.

\section{PCSK9 Inhibitors}

Proprotein convertase subtilisin/kexin type 9 (PCSK9) inhibitors have emerged as a novel class of medications for dramatic lowering of cholesterol levels. LDL receptors (LDLR) which are present on the liver and other cell membranes bind to and reduce serum LDL particles. PCSK9 enhances the degradation of liver LDLRs resulting in LDL accumulation. With PCSK9 blockade, more LDLRs on the surface of the cells become available which results in significant lowering of serum LDL levels. Individuals with loss-of-function mutations in PCSK9 have reduced levels of LDL cholesterol and therefore have been found to be protected from coronary heart disease. PCSK9 deficiency confers resistance to liver steatosis, but its effect on LDLR is independent of this function $[47,48]$.

At this time, the estimated cost-effectiveness of PCSK9 inhibitors outweigh the benefits and reducing the price remains the primary goal to improving the value of this therapy [49]. However, a recent randomized, double-blinded, placebocontrolled trial demonstrated when Evolocumab, a monoclonal antibody that inhibits PCSK9, was added to statin therapy, it lowered LDL cholesterol levels by $59 \%$, and it significantly reduced the risk of cardiovascular events with a $15 \%$ reduction 
in the risk of the primary composite endpoint of cardiovascular death, myocardial infarction, or stroke [50]. Current guidelines recommend PCSK9 inhibitors for patients who may not tolerate statins and for familial hypercholesterolemia [51]. The outcome of ongoing large scale cardiovascular endpoint trials testing PCSK9 inhibitors over next several years will be pivotal.

Circulating PCSK9 levels correlate with the severity of steatosis and increase with hepatic fat accumulation [52]. Theoretically, being a potent lipid lowering agent, PCSK9 inhibitors may decrease both the progression of NAFLD and decrease the associated cardiovascular risk. However, PCSK9 inhibitors are still novel, and we remain to have limited information about patient compliance and long term outcomes.

\section{Conclusion}

Health care providers should recognize the high prevalence of CVD in patients with NAFLD. The evidence demonstrated that statins were safe, lead to cardiovascular risk reduction and may potentially prevent the deterioration of NAFLD. In addition, with the great reduction in LDL cholesterol, PCSK9 inhibitors may prove to be an effective treatment for NAFLD patients. Further large scale prospective trials that show the long-term effects of statins or PCSK9 inhibitors are needed to change how health care providers manage and treat patients with NAFLD. Given the high prevalence of NAFLD in the general population, we suggest that the current guidelines add lipid lowering therapies to prevent CVD.

\section{References}

1. Rector RS, Thyfault JP, Wei Y, et al. Non-alcoholic fatty liver disease and the metabolic syndrome: an update. World J Gastroenterol. 2008;14:185-92.

2. Lazo M, Clark JM. The epidemiology of nonalcoholic fatty liver disease: a global perspective. Semin Liver Dis. 2008;28:339-50.

3. Byrne CD, Olufadi R, Bruce KD, et al. Metabolic disturbances in non-alcoholic fatty liver disease. Clin Sci (Lond). 2009;116:539-64.

4. McCullough AJ. Pathophysiology of nonalcoholic steatohepatitis. J Clin Gastroenterol. 2006;40:S17-29.

5. Hamaguchi M, Kojima T, Takeda N, et al. Nonalcoholic fatty liver disease is a novel predictor of cardiovascular disease. World J Gastroenterol. 2007;13:1579-84.

6. Soderberg C, Stal P, Askling J, et al. Decreased survival of subjects with elevated liver function tests during a 28-year follow-up. Hepatology. 2010;51:595-602.

7. Adams LA, Lymp JF, St Sauver J, et al. The natural history of nonalcoholic fatty liver disease: a populationbased cohort study. Gastroenterology. 2005;129:113-21.

8. Ekstedt M, Franzen LE, Mathiesen UL, et al. Long-term follow-up of patients with NAFLD and elevated liver enzymes. Hepatology. 2006;44:865-73.

9. Ruttmann E, Brant LJ, Concin H, et al. Gammaglutamyltransferase as a risk factor for cardiovascular disease mortality: an epidemiological investigation in a cohort of 163,944 Austrian adults. Circulation. 2005;112:2130-7.

10. Lee DH, Silventoinen K, Hu G, et al. Serum gammaglutamyltransferase predicts non-fatal myocardial infarction and fatal coronary heart disease among 28,838 middle-aged men and women. Eur Heart J. 2006;27:2170-6.

11. Fraser A, Harris R, Sattar N, et al. Gammaglutamyltransferase is associated with incident vascular events independently of alcohol intake: analysis of the British Women's Heart and Health Study and MetaAnalysis. Arterioscler Thromb Vasc Biol. 2007;27:2729-35.

12. Schindhelm RK, Dekker JM, Nijpels G, et al. Alanine aminotransferase predicts coronary heart disease events: a 10-year follow-up of the Hoorn Study. Atherosclerosis. 2007; 191:391-6.

13. Dunn $\mathrm{W}, \mathrm{Xu} \mathrm{R}$, Wingard $\mathrm{DL}$, et al. Suspected nonalcoholic fatty liver disease and mortality risk in a population-based cohort study. Am J Gastroenterol. 2008;103:2263-71.

14. Oni ET, Agatston AS, Blaha MJ, et al. A systematic review: burden and severity of subclinical cardiovascular disease among those with nonalcoholic fatty liver; should we care? Atherosclerosis. 2013;230:258-67.

15. Dekker JM, Girman C, Rhodes T, et al. Metabolic syndrome and 10-year cardiovascular disease risk in the Hoorn Study. Circulation. 2005;112:666-73.

16. Byrne CD, Targher G. Ectopic fat, insulin resistance, and nonalcoholic fatty liver disease: implications for cardiovascular disease. Arterioscler Thromb Vasc Biol. 2014;34:1155-61.

17. Anstee QM, Targher G, Day CP. Progression of NAFLD to diabetes mellitus, cardiovascular disease or cirrhosis. Nat Rev Gastroenterol Hepatol. 2013;10:330-44.

18. Targher G, Byrne CD, Lonardo A, et al. Non-alcoholic fatty liver disease and risk of incident cardiovascular disease: A meta-analysis. J Hepatol. 2016;65:589-600.

19. Byrne CD, Targher G. NAFLD: a multisystem disease. J Hepatol. 2015;62:S47-64.

20. Lee DH, Blomhoff R, Jacobs DR. Is serum gamma glutamyltransferase a marker of oxidative stress? Free Radic Res. 2004;38:535-9.

21. Chalasani N, Deeg MA, Crabb DW. Systemic levels of lipid peroxidation and its metabolic and dietary correlates in patients with nonalcoholic steatohepatitis. Am J Gastroenterol. 2004;99:1497-502.

22. Chen $\mathrm{CH}$, Nien $\mathrm{CK}$, Yang $\mathrm{CC}$, et al. Association between nonalcoholic fatty liver disease and coronary artery calcification. Dig Dis Sci. 2010;55:1752-60.

23. Akabame S, Hamaguchi M, Tomiyasu K, et al. Evaluation of vulnerable coronary plaques and non-alcoholic fatty liver disease (NAFLD) by 64-detector multislice computed tomography (MSCT). Circ J. 2008;72:618-25.

24. Assy N, Djibre A, Farah R, et al. Presence of coronary plaques in patients with nonalcoholic fatty liver disease. Radiology. 2010;254:393-400. 
Citation: Tarek A, Anuj C, Zachary O, et al. It's time to focus on decreasing cardiovascular mortality in NAFLD population: potential use of statins and PCSK9 inhibitors. Curr Trend Cardiol. 2017;1(2):\$\{pages\}.

25. Sung KC, Ryan MC, Wilson AM. The severity of nonalcoholic fatty liver disease is associated with increased cardiovascular risk in a large cohort of nonobese Asian subjects. Atherosclerosis. 2009;203:581-6.

26. Lavie CJ, Milani RV, Verma A, et al. C-reactive protein and cardiovascular diseases--is it ready for primetime? Am J Med Sci. 2009;338:486-92.

27. Wannamethee G, Ebrahim S, Shaper AG. Gammaglutamyltransferase: determinants and association with mortality from ischemic heart disease and all causes. Am J Epidemiol. 1995;142:699-708.

28. Witteles RM, Fowler MB. Insulin-resistant cardiomyopathy clinical evidence, mechanisms, and treatment options. J Am Coll Cardiol. 2008;51:93-102.

29. Peterson LR. Obesity and insulin resistance: effects on cardiac structure, function, and substrate metabolism. Curr Hypertens Rep. 2006;8(:451-6.

30. Bhatia LS, Curzen NP, Calder PC, et al. Non-alcoholic fatty liver disease: a new and important cardiovascular risk factor? Eur Heart J. 2012;33:1190-1200.

31. Fabbrini E, Magkos F, Mohammed BS, et al. Intrahepatic fat, not visceral fat, is linked with metabolic complications of obesity. Proc Natl Acad Sci U S A. 2009;106:15430-5.

32. Gastaldelli A, Kozakova M, Hojlund K, et al. Fatty liver is associated with insulin resistance, risk of coronary heart disease, and early atherosclerosis in a large European population. Hepatology. 2009;49:1537-44.

33. Volzke H, Robinson DM, Kleine V, et al. Hepatic steatosis is associated with an increased risk of carotid atherosclerosis. World J Gastroenterol. 2005;11:1848-53.

34. Targher G, Bertolini L, Padovani R, et al. Relations between carotid artery wall thickness and liver histology in subjects with nonalcoholic fatty liver disease. Diabetes Care. 2006;29:1325-30.

35. Fracanzani AL, Burdick L, Raselli S, et al. Carotid artery intima-media thickness in nonalcoholic fatty liver disease. Am J Med. 2008;121:72-8.

36. Sookoian S, Pirola CJ. Non-alcoholic fatty liver disease is strongly associated with carotid atherosclerosis: a systematic review. J Hepatol. 2008;49:600-7.

37. Kantartzis K, Rittig K, Cegan A, et al. Fatty liver is independently associated with alterations in circulating HDL2 and HDL3 subfractions. Diabetes Care. 2008;31:366-8.

38. Ratziu V, Bellentani S, Cortez-Pinto H, et al. A position statement on NAFLD/NASH based on the EASL 2009 special conference. J Hepatol. 2010;53:372-84.

39. Stone NJ, Robinson JG, Lichtenstein AH, et al. 2013 ACC/AHA guideline on the treatment of blood cholesterol to reduce atherosclerotic cardiovascular risk in adults: a report of the American College of Cardiology/American Heart Association Task Force on Practice Guidelines. Circulation. 2014;129:S1-45.

40. Vamecq J, Latruffe N. Medical significance of peroxisome proliferator-activated receptors. Lancet. 1999;354:141-8.
41. Sanguino E, Roglans N, Alegret M, et al. Atorvastatin reverses age-related reduction in rat hepatic PPARalpha and HNF-4. Br J Pharmacol. 2005;145:853-61.

42. Argo CK, Loria $\mathrm{P}$, Caldwell $\mathrm{SH}$, et al. Statins in liver disease: a molehill, an iceberg, or neither? Hepatology. 2008;48:662-9.

43. De Denus S, Spinler SA, Miller K, et al. Statins and liver toxicity: a meta-analysis. Pharmacotherapy. 2004;24:584-91.

44. Yoneda M, Mawatari H, Fujita K, et al. High-sensitivity Creactive protein is an independent clinical feature of nonalcoholic steatohepatitis (NASH) and also of the severity of fibrosis in NASH. J Gastroenterol. 2007;42:573-82.

45. Ekstedt M, Franzen LE, Mathiesen UL, et al. Statins in non-alcoholic fatty liver disease and chronically elevated liver enzymes: a histopathological follow-up study. J Hepatol. 2007;47:135-141.

46. Athyros VG, Tziomalos K, Gossios TD, et al. Safety and efficacy of long-term statin treatment for cardiovascular events in patients with coronary heart disease and abnormal liver tests in the Greek Atorvastatin and Coronary Heart Disease Evaluation (GREACE) Study: a post-hoc analysis. Lancet. 2010;376:1916-22.

47. Abifadel M, Varret M, Rabes JP, et al. Mutations in PCSK9 cause autosomal dominant hypercholesterolemia. Nat Genet. 2003;34:154-6.

48. Zaid A, Roubtsova A, Essalmani R, et al. Proprotein convertase subtilisin/kexin type 9 (PCSK9): hepatocytespecific low-density lipoprotein receptor degradation and critical role in mouse liver regeneration. Hepatology. 2008;48:646-54.

49. Kazi DS, Moran AE, Coxson PG, et al. Cost-effectiveness of PCSK9 Inhibitor Therapy in Patients With Heterozygous Familial Hypercholesterolemia or Atherosclerotic Cardiovascular Disease. JAMA. 2016;316:743-53.

50. Sabatine MS, Giugliano RP, Keech AC, et al. Evolocumab and Clinical Outcomes in Patients with Cardiovascular Disease. N Engl J Med. 2017.

51. Writing C, Lloyd-Jones DM, Morris PB, et al. 2016 ACC Expert Consensus Decision Pathway on the Role of NonStatin Therapies for LDL-Cholesterol Lowering in the Management of Atherosclerotic Cardiovascular Disease Risk: A Report of the American College of Cardiology Task Force on Clinical Expert Consensus Documents. J Am Coll Cardiol. 2016;68:92-125.

52. Ruscica M, Ferri N, Macchi C, et al. Liver fat accumulation is associated with circulating PCSK9. Ann Med. 2016;48:384-91.

\section{* Correspondence to}

Tarek Ajam

Department of Internal Medicine, Saint Louis University

Saint Louis, MO, USA

E-mail: tarekajam@gmail.com 
